OPEN ACCESS

Edited by:

Francesca Gioia Klinger, University of Rome Tor Vergata, Italy

Reviewed by: Juan Ge,

Qiangwang, China Jie Yan,

Peking University Third Hospital,

China

*Correspondence:

Qing-Yuan Sun

sunqy@gd2h.org.cn

Specialty section:

This article was submitted to Molecular and Cellular Reproduction,

a section of the journal

Frontiers in Cell and Developmental

Biology

Received: 08 May 2021

Accepted: 28 June 2021

Published: 26 July 2021

Citation:

Dong M-Z, Li Q-N, Fan L-H, Li L,

Shen W, Wang Z-B and Sun Q-Y

(2021) Diabetic Uterine Environment

Leads to Disorders in Metabolism

of Offspring.

Front. Cell Dev. Biol. 9:706879.

doi: 10.3389/fcell.2021.706879

\section{Diabetic Uterine Environment Leads to Disorders in Metabolism of Offspring}

\author{
Ming-Zhe Dong 1,2,3, Qian-Nan Li' ${ }^{2}$, Li-Hua Fan' ${ }^{2}$, Li Li ${ }^{2}$, Wei Shen ${ }^{1}$, Zhen-Bo Wang ${ }^{2,3}$ and \\ Qing-Yuan Sun ${ }^{4 *}$ \\ ${ }^{1}$ Institute of Reproductive Science, College of Life Sciences, Qingdao Agricultural University, Qingdao, China, ${ }^{2}$ State Key \\ Laboratory of Stem Cell and Reproductive Biology, Institute of Zoology, Chinese Academy of Sciences, Beijing, China, \\ ${ }^{3}$ University of Chinese Academy of Sciences, Beijing, China, ${ }^{4}$ Fertility Preservation Lab, Reproductive Medicine Center, \\ Guangdong Second Provincial General Hospital, Guangzhou, China
}

Aims: Research evidence indicates that epigenetic modifications of gametes in obese or diabetic parents may contribute to metabolic disorders in offspring. In the present study, we sought to address the effect of diabetic uterine environment on the offspring metabolism.

Methods: Type 2 diabetes mouse model was induced by high-fat diet combined with streptozotocin (STZ) administration. We maintained other effect factors constant and changed uterine environment by zygote transfers, and then determined and compared the offspring numbers, symptoms, body weight trajectories, and metabolism indices from different groups.

Result: We found that maternal type 2 diabetes mice had lower fertility and a higher dystocia rate, accompanying the increased risk of offspring malformations and death. Compared to only a pre-gestational exposure to hyperglycemia, exposure to hyperglycemia both pre- and during pregnancy resulted in offspring growth restriction and impaired metabolism in adulthood. But there was no significant difference between a pre-gestational exposure group and a no exposure group. The deleterious effects, no matter bodyweight or glucose tolerance, could be rescued by transferring the embryos from diabetic mothers into normal uterine environment.

Conclusion: Our data demonstrate that uterine environment of maternal diabetes makes critical impact on the offspring health.

Keywords: diabetes, uterus, metabolism, epigenetic inheritance, obese

\section{INTRODUCTION}

Diabetes is one of the largest global health emergencies of twenty-first century (Overland et al., 2014; Karuranga et al., 2019). The prevalence rate for obesity and type 2 diabetes has increased globally over recent decades at a pace. Maternal diabetes is a pathologic state that increases the incidence of complications in both the mother and the fetus (Vrachnis et al., 2012), for example, low quality oocytes (Zhang et al., 2013), increased chances of miscarriage (Mills et al., 1988) and congenital malformations of offspring (Vitoratos et al., 2010). Moreover, diabetic 
persons are considered to have significant positive relation for formation of hypercholesterolemia (Chowdhury and Nessa, 2019) and high LDL cholesterol (Jayakumari et al., 2020). Previous study has demonstrated that epigenetic inheritance via gametes by itself could increase an offspring's susceptibility to develop to obesity and type 2 diabetes (Huypens et al., 2016). Specifically, we have found that paternal prediabetes increases the susceptibility to diabetes in offspring through gametic epigenetic alterations (Wei et al., 2014). The epigenetic profiling of pancreatic islets was changed in offspring depending on paternal prediabetes (Wei et al., 2014). Another study shows that sperm tsRNAs represent a paternal epigenetic factor that may mediate intergenerational inheritance of diet-induced metabolic disorders (Chen et al., 2016). Injection of sperm tsRNA fractions from high fat diet (HFD)-fed males into normal zygotes generated metabolic disorders in the F1 offspring (Chen et al., 2016). On the other hand, other studies show that gestational diabetes may have effects on offspring (Fetita et al., 2006; Sasson et al., 2015). An exposure to HFD only during gestation resulted in fetal growth restriction and decreased placental weight (Fetita et al., 2006; Sasson et al., 2015). We still know little about the effects of maternal diabetes on the growth and metabolism of offspring and how to change this kind of susceptibility to prevent this destructive cycle of metabolic dysfunction through generations.

Although the adverse effects of diabetic mothers on offspring may be owing to the epigenetic modifications in oocytes of diabetic mothers, the poor intrauterine environment may also play important roles. Previous studies have shown that offspring of gestational obese mothers or mothers fed a high-fat diet during gestation not only exhibited metabolic disorders but also impaired skeletal muscle development (Levin and Govek, 1998; Bayol et al., 2005; Huang et al., 2017). In our previous study, we showed that although embryo development was adversely affected by maternal diabetes, no evident imprinting abnormality was observed in oocytes from female offspring derived from a diabetic mother, and methylation in offspring's oocytes is normal (Ge et al., 2013b). We further showed that the effects of maternal diabetes on imprinted genes might primarily be caused by the adverse uterus environment (Ge et al., 2013a). We do not clearly know which reason, uterus environment or gamete epigenetic inheritance, could be more important to increase offspring's susceptibility to obesity or type 2 diabetes. To this end, we successfully generated a severe type 2 diabetic mouse model, and by zygote transfer demonstrated that uterine environment of maternal diabetes makes more impact on the offspring compared with epigenetic inheritance via gametes.

\section{MATERIALS AND METHODS}

Male and female C57BL/6J mice provided by SPF (Beijing) Biotechnology Co. Ltd. were housed in ventilated cages on a 12$\mathrm{h}$ light/12-h dark cycle at a constant temperature $\left(23 \pm 1^{\circ} \mathrm{C}\right)$ and under controlled humidity $(60 \pm 5 \%)$. Mice were ad libitum fed water and food. All mouse experiments were approved by the Ethics Committee of the Institute of Zoology, Chinese Academy of Sciences.

\section{Diabetic Mouse Model}

We generated a type 2 diabetes mouse model according to previous studies, with slight changes (Wei et al., 2014). In brief, 3 weeks old C57BL/6J mice, five per cage, were randomly divided into two groups and fed with either a HFD (D12492; Research Diet, New Brunswick, NJ, United States) or a normal standard chow (NC, SPF Rodent Growth and Breeding Feed, BEIJING KEAO XIELI FEED CO., LTD) until 20 weeks of age. During 1215 weeks old, mice fed with HFD diet received intraperitoneal injection of a sub-diabetogenic dose of STZ $(100 \mathrm{mg} / \mathrm{kg}$ body weight, S0130, Sigma) once a week, a total of 4 times, and kept on the same diet until 20 weeks old. The fasting plasma glucose was measured after overnight fasting and the casual plasma glucose was measured at any point in time by a glucometer (Accu-CHEK active; Roche Diagnostic). The measurement was executed every week between 17 and 20 weeks of age after STZ injection. Mice with a casual plasma glucose $\geq 200 \mathrm{mg} / \mathrm{dl}(11.1 \mathrm{mmol} / \mathrm{l})$ or a fasting plasma glucose $\geq 126 \mathrm{mg} / \mathrm{dl}(7.0 \mathrm{mmol} / \mathrm{l})$ were considered as having diabetes.

\section{Experimental Design}

To test the impact of uterine environment on metabolism of offspring, female type 2 diabetes mice (hereafter called $\mathrm{DP}_{0}$ ) about 21 weeks old were mated with normal C57BL/6J males for generating offspring $\left(\mathrm{DCF}_{1}\right.$ and $\left.\mathrm{DF}_{1}\right)$ from different uterine environment by transferring the zygotes into healthy mature foster ICR (Institute of Cancer Research) mothers or no transferring (Figure 1). On the other hand, to test whether the metabolism of offspring could be affected by gamete epigenetic inheritance or not, the zygotes of female normal diet control mice (hereafter called $\mathrm{CP}_{0}$ ) as same age as $\mathrm{DP}_{0}$ mated with normal C57BL/6J males were also transferred into healthy foster ICR mothers to generate offspring $\left(\mathrm{CCF}_{1}\right)$ which were compared with $\mathrm{DCF}_{1}$ (Figure 1). Each foster mother would receive 6-12 zygotes. The number of litters per group was not less than 4 . All offspring were breast fed by ICR mothers and subjected to normal standard chow after weaning. Each ICR mothers feed pups no more than 6 to ensure enough milk. We also designed an untreated group, in which the normal maternal C57BL/6J mice were mated with normal C57BL/6J males with no zygotes transfer to analyze the reproductive phenotype as control. These offspring of $\mathrm{CP}_{0}$ is named $\mathrm{CF}_{1}$.

\section{Zygote Collection and Offspring Production}

Zygote collection and transfer were conducted following standardized procedures described in the Manipulating the Mouse Embryos. In brief, C57BL/6J females (HFD and NC) in heat were mated with normal C57BL/6J males between 20 and 22 weeks of age, and zygotes were collected from a part of the successfully mated female mice. The collected zygotes of each female mouse were analyzed and subsequently used for oviduct transfer into ICR recipient mother on normal diet. Each foster mother would receive 6-17 zygotes which were divided into two parts and transferred into the bilateral oviducts. The birth rate was the ratio of birth number to the 


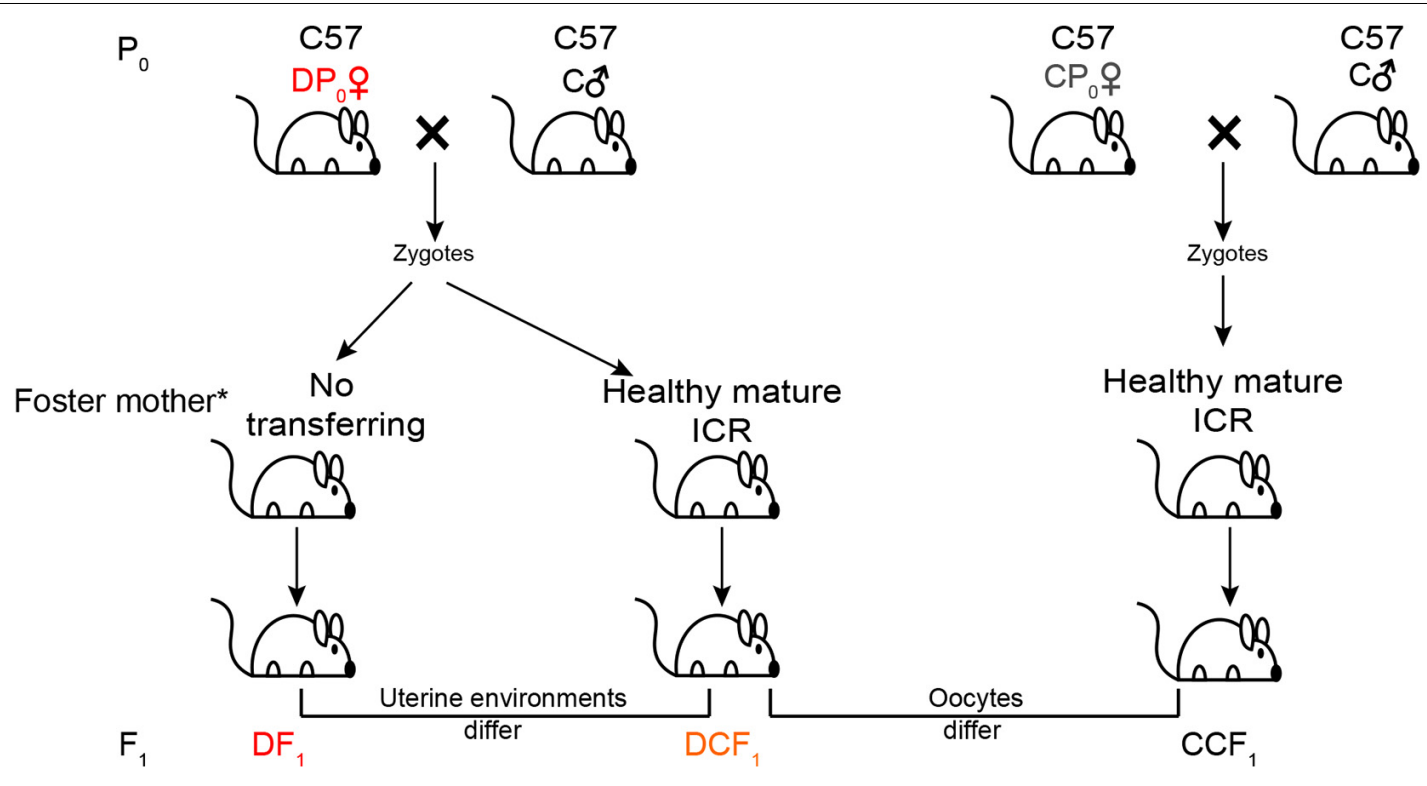

FIGURE 1 | Experimental design. Maternal C57BL/6J mice $\left(\mathrm{P}_{0}\right)$ were at random allocated to induce or not induce diabetes. The diabetic mice (DP0) and non-diabetic mice $\left(\mathrm{CP}_{0}\right)$ were mated with normal C57BL/6J males $\left(\mathrm{C} \sigma^{7}\right)$ and their zygotes were collected and transferred into the oviduct of healthy mature ICR foster mother. For the other part of successfully mated $\mathrm{DP}_{0}$ mice, their zygotes were not transferred and incubated in the original diabetic uterus of $\mathrm{DP}_{0}$. Newborn offspring $\left(F_{1}\right)$ of these three group were all fed by ICR foster mother. All offspring were weaned at 3 weeks of age and placed on a normal diet. *All offspring were breast fed by ICR mothers.

number of embryos transferred. $\mathrm{F}_{1}$ of the two groups $\left(\mathrm{DCF}_{1}\right.$ and $\mathrm{CCF}_{1}$ ) were all fed by ICR mice during lactation. $\mathrm{DF}_{1}$ mice were generated by another part of the successfully mated female HFD mice and fed by ICR mice during lactation in an identical fashion.

\section{Dystocia Rate and Cesarean Section}

If the pregnant female mouse could not attempt the vaginal delivery at day 21 of pregnancy, it was classified as dystocia and recorded. The dystocia rate was the ratio of dystocia number to the number of all pregnancy in each group. Then cesarean section was conducted right after cervical dislocation by hysterectomy, and pups were quickly wiped clean and placed on a warming plate at $37^{\circ} \mathrm{C}$. Before transferring to a foster mother, the survival number and abnormal number of each litter were recorded. For the vaginal delivery pups, the survival number and abnormal number of each litter were recorded at the first day they were born. The survival rate of each group was worked out by dividing the survival number by the total birth number. However, the abnormal rate of each group was worked out by dividing the abnormal number after weaning by the total birth number, because some malformations in eye was diagnosed until weaning. A minimum of 4 litters were used in each group.

\section{Body Weight Measurement}

The body weights of maternal mice were measured every week between 7 and 21 weeks of age. The newborns' weights were measured at the 1 day of age. Then, we weighed the offspring every week from 3 to 15 weeks of age.

\section{ITT and GTT}

ITT (insulin tolerance test) and GTT (glucose tolerance test) were performed as previously described (Wei et al., 2014) and the offspring of each group used for the glucose tolerance were from different litters. In brief, ITT was conducted after a fasting period of $2 \mathrm{~h}$. Mice received 1 unit of insulin (I-5500, Sigma) per kilogram of body weight by intraperitoneal injection. The concentration of insulin solution used for injection was 0.05 $\mathrm{U} / \mathrm{ml}$. Blood glucose levels from tail blood were determined at $0,15,30,60$, and $120 \mathrm{~min}$ after application of the insulin by a glucometer. GTT was conducted after an overnight fasting period of $13 \pm 1 \mathrm{~h}$. Mice received $2 \mathrm{~g}$ of glucose per kilogram of body weight by intraperitoneal injection. Glucose was dissolved in water to $10 \%$ (mass-to-volume) concentration and used to inject. Blood glucose levels from tail blood were determined at $0,15,30$, 60 , and $120 \mathrm{~min}$ after application of the glucose challenge by the same glucometer. Offspring of different litters (no less than 4 in each group) were randomly selected and analyzed for the test.

\section{Serum Biomarkers Test}

Serum was isolated from blood sampled by eyeball extirpating and stored at $-80^{\circ} \mathrm{C}$ until detection. HuNan FengRui Biotechnology Co., Ltd. was employed for biochemical detection of serum. The tested items include the total cholesterol (TC), the triglyceride (TG), high-density lipoprotein cholesterol (HDL) and low-density lipoprotein cholesterol (LDL).

\section{Statistical Analysis}

All analyses were performed using GraphPad Prism 5 software. Results were expressed as mean \pm S.E.M. For all comparisons that 
involved multiple time points, a two-way repeated measurement ANOVA, post hoc Bonferroni multiple-comparisons test was used to assign $P$-values by comparing to each other in the F1 generation. In the P0 generation, $\mathrm{CP} 0$ o served as control group. For measurements at single time points, a one way ANOVA was used. To assess differences in blood glucose of P0 generation, post hoc Bonferroni multiple-comparisons test was applied to assign $P$-values vs. the control group. To assess differences in body weight distribution in F1 cohorts, a Kruskal-Wallis test, using Dunn's multiple-comparisons test, was applied as a nonparametric test to assign $P$-values. The D'Agostino and Pearson omnibus normality test was applied to assess the survival rate and abnormality rate of F1 in each litter. A two-tailed Student's $\mathrm{t}$ test was performed to compare two different groups, and an unpaired test was performed on non-parametric data $P<0.05$ was considered statistically significant.

\section{RESULTS}

\section{Establishment of the Diabetes Mouse Model}

To explore which has more effects on the offspring health of diabetes, uterine environment or oocytes, we generated type 2 diabetes mouse model by feeding high fat diet combined with STZ, a diabetagen which is especially toxic to pancreatic islet insulin-producing $\beta$-cells, injection at a low dose. The body weight of high fat diet type 2 diabetes mice $\left(\mathrm{DP}_{0}\right)$ was significantly higher than that of normal diet control mice $\left(\mathrm{CP}_{0}\right)$, when measured between 7 and 21 weeks of age. However, the body weight of $\mathrm{DP}_{0}$ was increased before 19 weeks of age, and subsequently decreased in 3 weeks after STZ injection (Figure 2A). According the diagnostic criteria for diabetes mentioned in the materials and methods, we picked 34 mice which successfully developed diabetes from 50 suffering treatment for the following research. Sixteen mice which did not develop diabetes were excluded from the study. The fasting plasma glucose and the casual plasma glucose during pregestational period, as well as the casual plasma glucose during gestational period of $\mathrm{DP}_{0}$ were all significantly higher than in $\mathrm{CP}_{0}$ mice (Figures $2 \mathrm{~B}-\mathrm{D}, 7.61 \pm 0.41$ vs. $4.2 \pm 0.06,17.33 \pm 0.9$ vs. $8.13 \pm 0.2,14.97 \pm 1.25$ vs. $8.29 \pm 0.25$, respectively, $P<$ $0.001)$. Next, we analyzed insulin resistance of the female mice in both $\mathrm{DP}_{0}$ and $\mathrm{CP}_{0}$ when they were 21 weeks of age. The mice of $\mathrm{DP}_{0}$ showed decreased insulin sensitivity compared with controls (Figure 2E). On the other hand, because the plasma glucose in GTT was too high to be measured, data of GTT was not shown.

\section{Female Diabetic Mice Had Reduced Fertility, Accompanying the Increased Risk of Offspring Malformations and Death, Which Could Be Rescued by Zygote Transfer}

Female mice, $\mathrm{DP}_{0}$ or $\mathrm{CP}_{0}$, were mated with normal diet control male mice in which the fasting and casual plasma glucose were both normal (Figures 2B,C). The natural ovulation of per female mouse was counted when a vaginal plug was observed next morning. The number of natural ovulation was significantly lower in the diabetic group than control group by 20 weeks of age (Figure 3A, $7 \pm 0.68$ vs. $11 \pm 0.4, P<0.01$ ). Associated with the decrease in natural ovulation, $\mathrm{DP}_{0}$ female mice become significantly low reproductive, with the offspring number of $\mathrm{DP}_{0}$ female mice decreased about 1.6-fold compared with the $\mathrm{CP}_{0}$ group (Figure 3B, $5.17 \pm 0.51$ vs. $8.25 \pm 0.41, P<0.01$ ). The dystocia rate of $\mathrm{DP}_{0}$ female mice also was very high compared with $\mathrm{CP}_{0}$ female mice (Figure 3C, 88 and $0 \%$, respectively).

Meanwhile, the survival rate of offspring, $\mathrm{DF}_{1}$ exposed to diabetic uterine, was decreased to only $38 \%$ compared to $\mathrm{CF}_{1}$ (Figure 3D). However, we did not observe differences in survival rate and birth rate between the two transfer groups, $\mathrm{DCF}_{1}$ and $\mathrm{CCF}_{1}$, when zygotes of $\mathrm{DP}_{0}$ mice or $\mathrm{CP}_{0}$ mice were collected and transferred into control ICR recipients (Figures 3D,E). The offspring exposed to maternal diabetes during gestation also manifested severe congenital malformation vs. offspring of control, with the abnormality rate of $18 \%$ (Figure $3 F$ ) in $\mathrm{DF}_{1}$ group. The congenital malformation includes neck hypertrophy and eye diseases (anophthalmia, microphthalmia, and white eye disease) (Figure 3G). There was also no congenital malformation being detected in the offspring when the zygotes were transferred into normal uterine environment (Figure 3F).

\section{Diabetic Uterine Environment Caused Lower Birth Weight and Growth Restriction After Birth}

To further explore the effect of diabetic uterine environment to the offspring, we analyzed the weight between $\mathrm{DF}_{1}, \mathrm{DCF}_{1}$, and $\mathrm{CCF}_{1}$. As shown in Figure 4A, DF 1 mice had lower birth weight than $\mathrm{DCF}_{1}(0.90 \pm 0.02$ vs. $1.32 \pm 0.01, P<0.001)$, while there was no significant difference between $\mathrm{DCF}_{1}$ and $\mathrm{CCF}_{1}(1.32 \pm 0.01$ vs. $1.33 \pm 0.01, P>0.05)$. Then the pups of all three groups were fed by ICR mice until to weaning. After weaning, the three groups were all fed normal standard chow and the body weight was recorded from 3 to 15 weeks old. Female offspring from diabetic mother and diabetic uterine environment, $\mathrm{DF}_{1}$, , demonstrated growth restriction compared with $\mathrm{DCF}_{1}$ and $\mathrm{CCF}_{1}$ oespecially at 4 and 14 weeks of age, showing significantly different (Figure 4B). However, there was no significant difference in body weight between $\mathrm{DCF}_{1}$ and $\mathrm{CCF}_{1}$ ( $($ Figure 4B). The distributions of body weights in male $\mathrm{F}_{1}$ cohorts of the three groups demonstrated the corresponding curve. What was different was that the growth restriction of $\mathrm{DF}_{1}$ $\sigma^{7}$ was more significant compared to $\mathrm{DCF}_{1} \mathrm{\sigma}^{7}$ and $\mathrm{CCF}_{1} \mathrm{o}^{7}$ from 3 to 15 weeks of age (Figure 4C). However, there was no significant difference in body weight between $\mathrm{DCF}_{1}$ and $\mathrm{CCF}_{1}$ 우 (Figure 4C).

\section{DF $_{1}$ Exhibited Impaired Metabolism, While the Metabolism of DCF $_{1}$ Was Normal}

To investigate whether the metabolism of offspring was affected by uterine environment or oocytes of maternal diabetes, we analyzed glucose tolerance and insulin tolerance in all the 

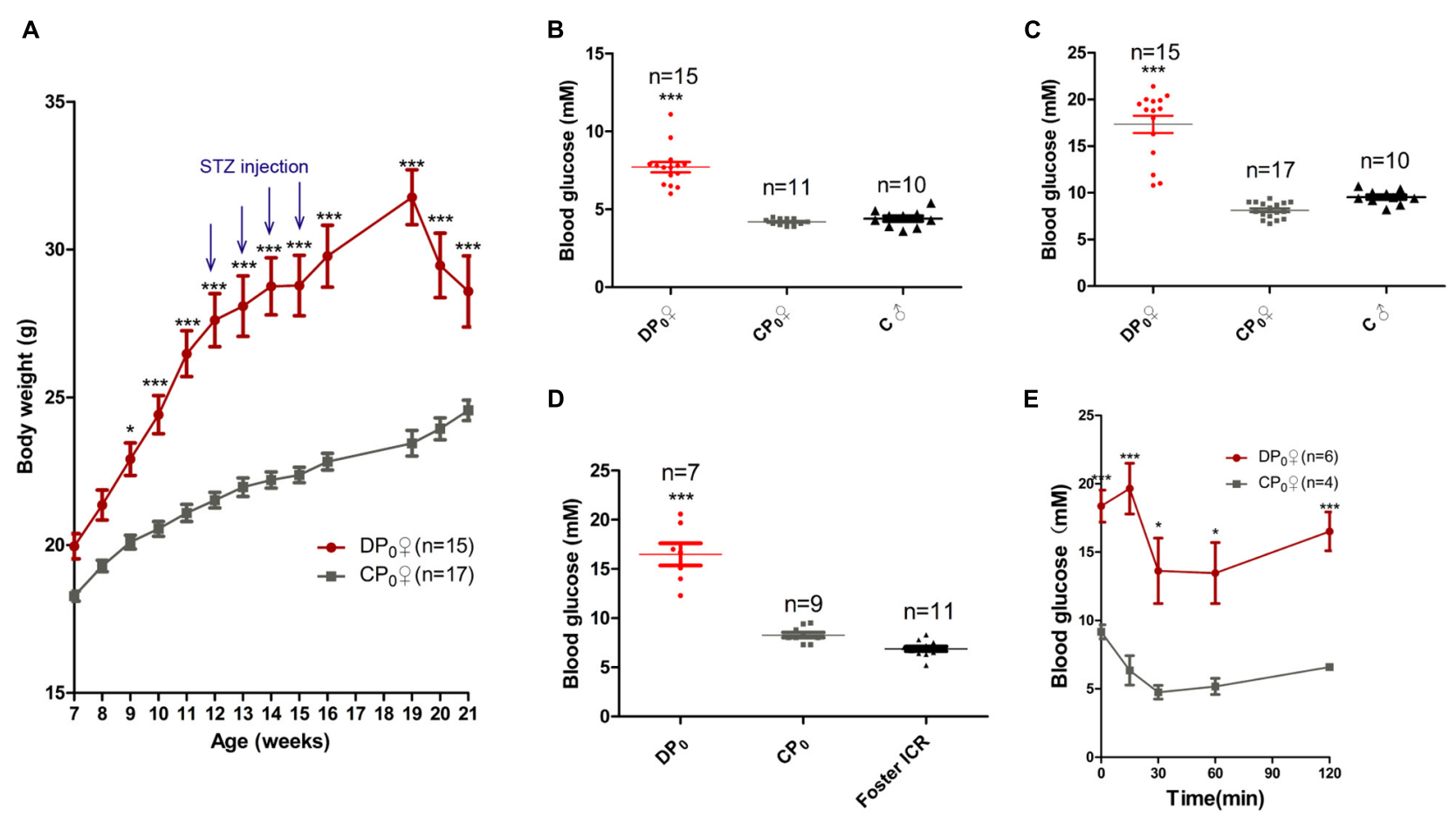

FIGURE 2 | Maternal $\left(P_{0}\right)$ metabolic phenotype. (A) Body weight gain from 7 to 21 weeks. (B) Fasting plasma glucose. (C) Casual plasma glucose. (D) Gestational plasma glucose. (E) ITT at 21 weeks of age. Data are expressed as mean \pm S.E.M.; ${ }^{*} P<0.05,{ }^{\star \star \star} P<0.001$ vs. $\mathrm{CP}_{0}$ control. A two-way repeated measurement ANOVA in (A,E) and a one way ANOVA in (B-D), post hoc Bonferroni multiple-comparisons test was used to assign $P$-values. $P$-values for significance between groups in repeated measure analysis are shown.

three $F_{1}$ cohorts when they were 16 weeks old. As shown in Figure 5A, the blood glucose levels during an intraperitoneal glucose tolerance test (GTT) were not significantly different among the female mice of the three groups. But, DF1 females showed insulin resistance in the ITT when they were 16 weeks old (Figure 5B). To the three male $F_{1}$ cohorts, there was no significant difference in either GTT or ITT when they were 16 weeks old (Figures 5C,D). We continued to feed the three male $F_{1}$ cohorts by normal diet until 1 year of age, which was equivalent to around 42.5 years old of human. As shown in Figure 5E, there was no significant difference in the body weight of the three groups. Then, we analyzed glucose tolerance in these middle age mice. Interestingly, $\mathrm{DF}_{1}$ males displayed an impaired glucose tolerance vs. $\mathrm{DCF}_{1}$ or $\mathrm{CCF}_{1}$ at this life phase (Figure 5F). However, $\mathrm{DCF}_{1}$ displayed a normal glucose tolerance vs. $\mathrm{CCF}_{1}$ (Figure 5F). We also analyzed the insulin tolerance when they were 45 weeks of age, but there was no significant difference among the three groups (Figure 5G).

\section{DISCUSSION}

People with diabetes are at higher risk of developing a number of disabling and life-threatening health problems than people without diabetes (Overland et al., 2014), such as diabetic eye disease, diabetic nephropathy and diabetic foot disease (Abuaisha et al., 1998; Apelqvist et al., 2000; Calvo-Maroto et al., 2014).
In particular, women with any type of diabetes are at risk of a number of complications during pregnancy, as high glucose levels can affect the development of the fetus (Overland et al., 2014). However, we did not clearly know which factor, the oocytes from diabetes or diabetic uterine environment, plays the crucial role in the development of their offspring.

Maternal diabetes may induce delayed oocyte maturation, abnormal cellular metabolism, mitochondrial dysfunction and meiotic defects (Ratchford et al., 2007; Wang et al., 2009, 2010; Cheng et al., 2011). It is well known that oocytes are deficient in their ability to use glucose as an energy substrate and require cumulus cells to provide products of glycolysis for their own development (Sugiura and Eppig, 2005). Maternal diabetes may indirectly impair oocyte competence by disrupting mitochondrial function in cumulus cells and their communications with the oocytes (Wang et al., 2010), which could have an impact on the provision of essential nutrients or information molecules. In our present study, we also demonstrated reduced natural ovulation (Figure 3A) and lower reproductive ability (Figure 3B). In addition, oocyte development and maturation are usually accompanied by the establishment of maternal imprints (Saitou et al., 2012). Some imprinted genes appear to be involved in lipid and energy metabolism (Haig, 2004), and even affect postnatal metabolism (Millership et al., 2019). Previously, we found that maternal diabetes not only causes the alterations of DNA methylation statuses of some imprinted genes in maternal oocytes (Ge et al., 2013b), but also led to the alterations of the 
A

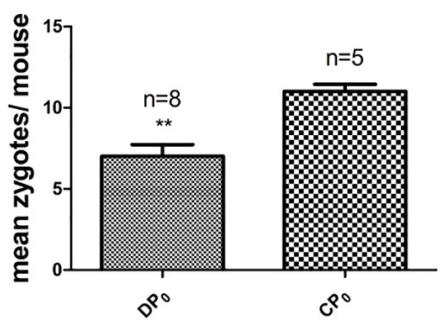

D

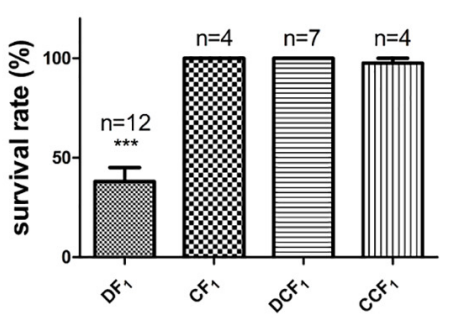

B

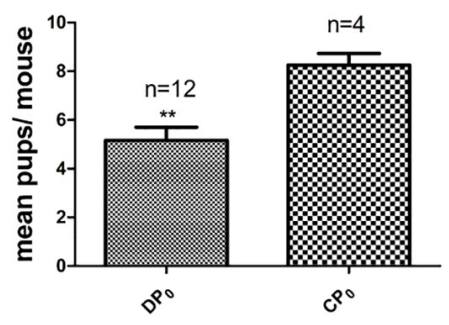

E

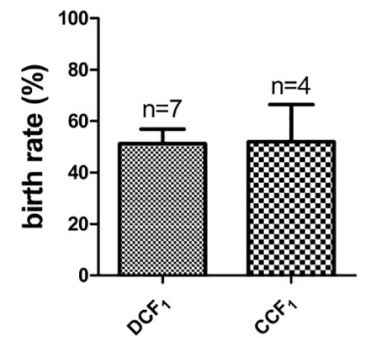

C

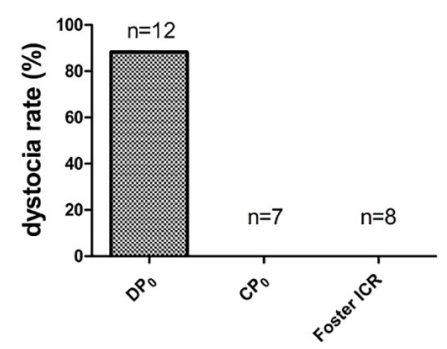

$\mathbf{F}$

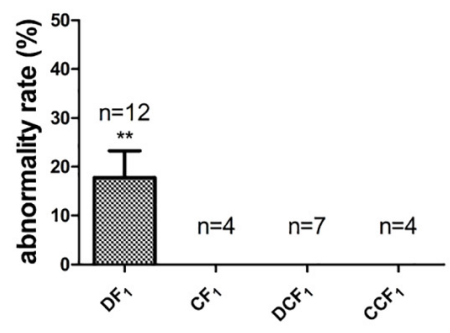

G

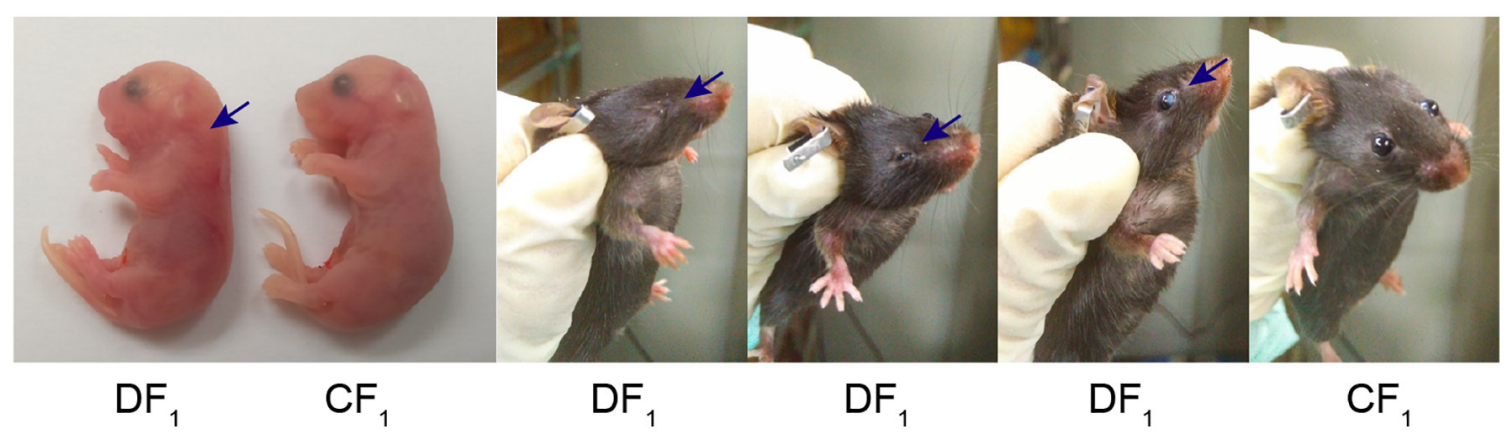

FIGURE 3 | Maternal reproductive phenotypes and offspring symptoms. (A) The number of zygotes per mouse. (B) The number of pups per mouse. (C) The maternal dystocia rate. (D) Survival rate of $F_{1}$ in each group. (E) Birth rate of DCF1 and $C C F_{1}$. (F) Abnormality rate of $F_{1}$ in each group. (G) Congenital malformations of $\mathrm{DF}_{1}$ compared to $\mathrm{CF}_{1}$. Blue arrows show abnormal regions. Data are expressed as mean \pm S.E.M.; ${ }^{* \star} P<0.01,{ }^{* \star *} P<0.001$ vs. $\mathrm{CP}_{0}$ or $\mathrm{CF}_{1}$ control. $\mathrm{A}$ two-tailed Student's $t$-test was performed in (A,B,E). The D'Agostino and Pearson omnibus normality test was applied in (D,F). $P$-values for significance between groups in repeated measure analysis are shown.

methylation patterns and expressions of the imprinted genes in mid-gestational placental tissues (Ge et al., 2013a).

On the other hand, the adverse uterine environment of maternal diabetes could cause an increase in placenta weight and elevated glucose levels in both plasma and amniotic fluid (Laurino et al., 2019). In the diabetes, higher placental weights showed reduced placental efficiency and inability to transfer nutrients to the developing fetus (Volpato et al., 2015). In addition to the placental influence, the high rate of glucose in the amniotic fluid can prolonged the fetuses $\beta$-pancreatic cells stimulation, causing a pancreatic insulin depletion and hypoinsulinemia (Lopez-Soldado and Herrera, 2003). At the crucial period of epigenetic reprogramming, adverse uterine environment may influence the establishment and maintenance of methylation in mice. For example, Martino et al. reported that placental mRNA abundance for the folate receptor alpha (FOLR1) and DNA methyltransferases (DNMT1) were influenced with raised BMI (Martino et al., 2018). Although the mechanisms remain to be clarified, the alterations of the methylation patterns and expressions of the imprinted genes in mid-gestational placental tissues (Ge et al., 2013a) may influence the postnatal metabolism of offspring (Crespi, 2019).

In the present study, we generated the type 2 diabetes mouse model, which exhibited the symptoms of overweight, high level fasting plasma glucose and insulin resistance (Figures 2A,B,E). In addition, the casual plasma glucose and gestational plasma glucose were abnormally high as well (Figures 2C,D). To compare the effect of uterine environment and oocytes on offspring, we analyzed the symptoms of the offspring from diabetic uterine environment or not by changing the single variable factor. As shown in the results, DF1 mice, offspring from diabetic uterine environment, exhibited lower survival rate and higher malformation rate (Figures $3 \mathrm{D}, \mathrm{F}$ ) than $\mathrm{DCF}_{1}$ mice, under the premise of no difference in embryo transplantation 

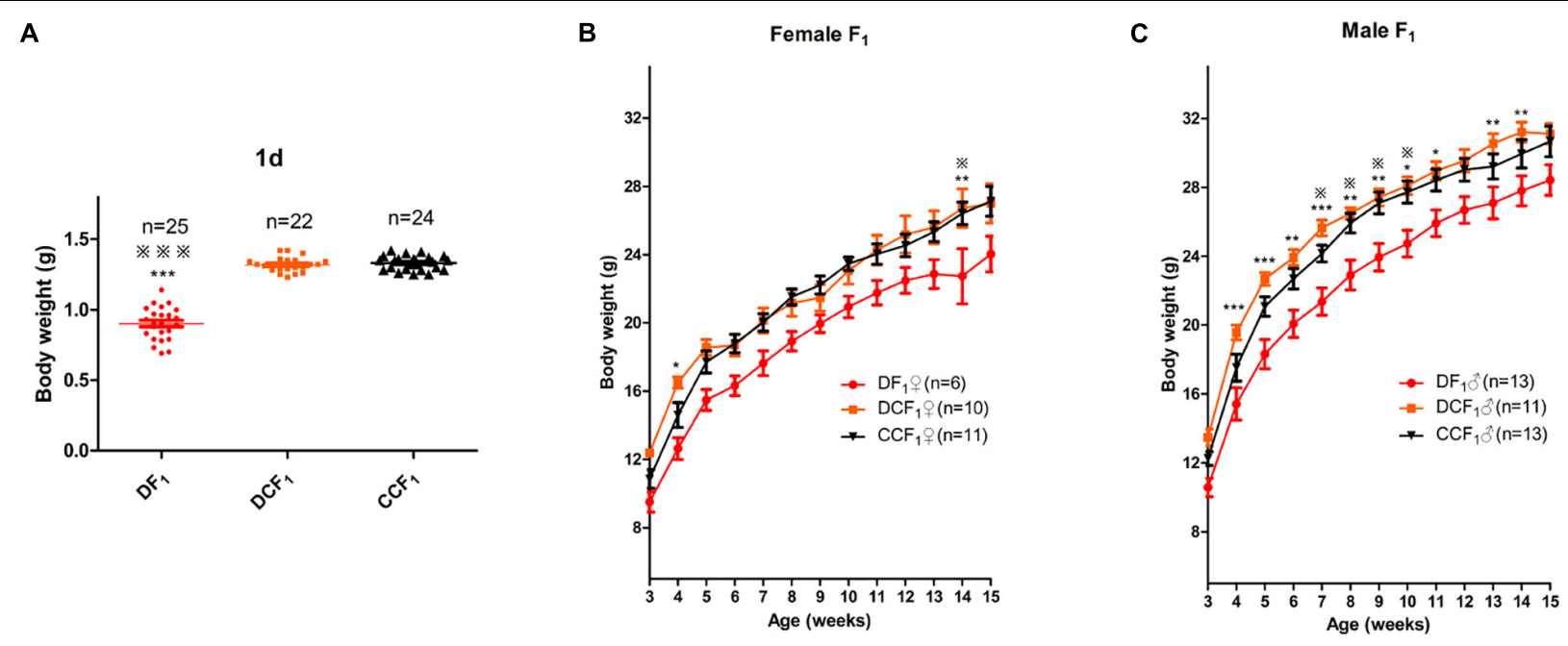

FIGURE $4 \mid F_{1}$ body weight distributions and trajectories. (A) Scatter plots of the body weight distribution for $F_{1}$ at 1 day of age. (B,C) The body weight trajectories of female $\mathrm{F}_{1} \mathbf{( B )}$ and male $\mathrm{F}_{1} \mathbf{( C )}$ mice between 3 and 15 weeks of age. Data are expressed as mean $\pm \mathrm{S} . \mathrm{E} . \mathrm{M}$.; ${ }^{\star} P<0.05$, ${ }^{\star \star} P<0.01$, ${ }^{\star \star \star} P<0.001 \mathrm{DF}_{1}$ Vs. $\mathrm{DCF}$. . Statistical significance, $\mathrm{DF}_{1}$ vs. $\mathrm{CCF}_{1}$, is represented using $\%$. There is no statistical significance between DCF 1 and CCF1. A one way ANOVA in (A) and a two-way repeated measurement ANOVA in $\mathbf{( B , C )}$, post hoc Bonferroni multiple-comparisons test was used to assign $P$-values by comparing to each other. $P$-values for significance between groups in repeated measure analysis are shown.

(Figure 3E). The malformation included neck hypertrophy during fetus and eye disease after birth (Figure 3G), which caused by the adverse uterine environment. For the maternal diabetes, the number of natural ovulation and dystocia rate both suffered adverse effect (Figures 3A,C), just like human beings with gestational diabetes (Overland et al., 2014). It was worth mentioning that our diabetic model had a far higher level of dystocia rate than human beings, which may be related to the severity of maternal diabetes. Because the female diabetic mice in our research had caught severe diabetes when they were pregnant which could be judged by the loss of their weight and the high gestational plasma glucose (Figures 2A,D).

In addition, gestational diabetes mellitus (GDM) and obesity are both complications which occur during pregnancy and subsequently influence the development of offspring during fetal life and postnatal development (Yessoufou and Moutairou, 2011). In our analysis, $\mathrm{DF}_{1}$ showed low birth weight and growth restriction after birth (Figure 4A), yet there was no significant difference between $\mathrm{DCF}_{1}$ and $\mathrm{CCF}_{1}$ when they were subjected to a normal diet (Figure 4). However $\mathrm{DF}_{1}$ displayed catch-up growth and the difference of weight among the three groups was gone until 16 weeks old (Figures 4B,C). We also examined the total cholesterol (TC), high-density lipoprotein cholesterol (HDL), low-density lipoprotein cholesterol (LDL), and the triglyceride (TG) of parents and offspring. For $\mathrm{DP}_{0}$, they had elevated $\mathrm{HDL}$ and $\mathrm{TC}$ compared with $\mathrm{CP}_{0}$, which may be responsible for the decreased body weight (Tan, 1986) and the hypercholesterolemia, although there were no significant difference in LDL and TG (Supplementary Figure 1). However, the hypercholesterolemia had not been passed on to the next generation, because there is no significant difference among $\mathrm{DF}_{1}, \mathrm{DCF}_{1}$, and $\mathrm{CCF}_{1}$ when they were 16 weeks old. It is possible that there are other metabolic abnormalities such as impaired beta-cell function, energy expenditure, physical activity or behavior changes that characterize these offspring (Sasson et al., 2015). It is clear that the risk of becoming diabetic is greater for relatives of diabetics than for individuals of the same age and sex in the general population (Simpson, 1968). Besides genetic factors, the offspring's risk of developing NIDDM is influenced by environmental factors, especially obesity (Pierce et al., 1995). Type 2 diabetes is one form of diabetes, in which the insulin resistance of patients is mostly caused or aggravated by obesity (Alberti and Zimmet, 1998). Interestingly, no matter maternal undernutrition (Schulz, 2010; Hales and Barker, 2013) or maternal hyper-nutrition, e.g., obesity and maternal diabetes (Lowe et al., 2018; Hod et al., 2019), the offspring has a greatly increased susceptibility to the development of type 2 diabetes. For the undernutrition model, the thrifty phenotype hypothesis was proposed to explain the effects of poor nutrition in fetal stage to subsequent metabolic syndrome (Hales and Barker, 2001). However, contextually with the dramatic spread of obesity and type 2 diabetes, the prevalence of GDM has significantly increased over the last few years (Zhu and Zhang, 2016; Filardi et al., 2019). The prevalence of GDM has increased by more than $30 \%$ within one or two decades in a number of countries including developing countries, forming an emerging worldwide epidemic (Guariguata et al., 2014; Zhu and Zhang, 2016).

In our present study, the offspring from diabetic uterine environment showed impaired metabolism and was recovered by transfer into normal uterus (Figure 5). In addition, the metabolic phenotypes displayed difference between sexes. As shown in Figure 5, female DF1 developed metabolic disorder earlier than male, as male DF1 displayed an impaired glucose 

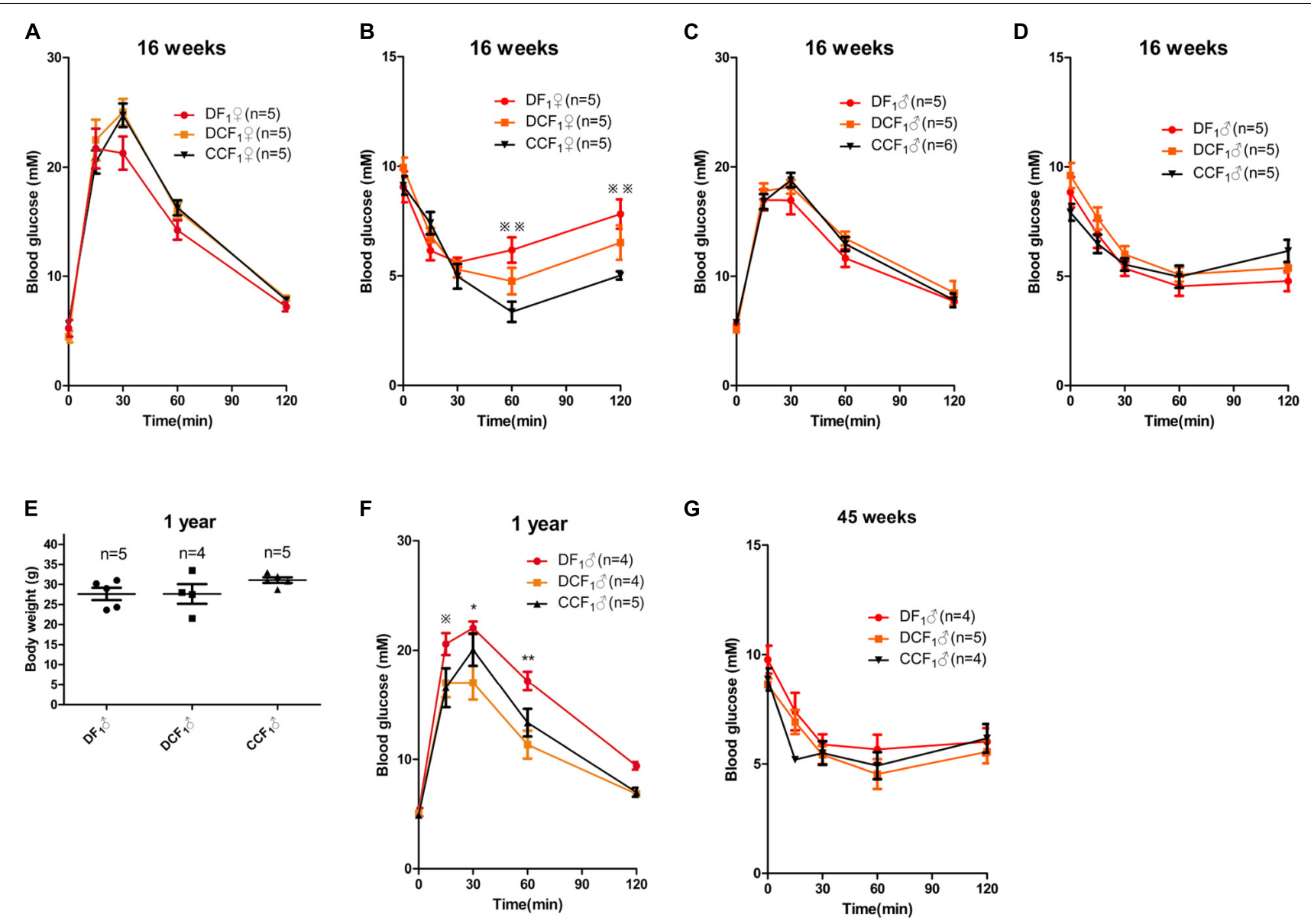

FIGURE 5 $\mid F_{1}$ metabolic phenotype. (A,C) Blood glucose concentrations during GTT in female (A) and male (C) $F_{1}$ mice at 0 , $15,30,60$, and 120 min after intraperitoneal glucose injection when they were 16 weeks of age. (B,D) Blood glucose concentrations during ITT in female (B) and male (D) $F_{1}$ mice at 0,15 , 30 , 60 , and 120 min after intraperitoneal insulin injection when they were 16 weeks of age. (E) Scatter plots of the body weight distribution for male $F_{1}$ at 1 year of age. (F) Blood glucose concentrations during GTT in male $F_{1}$ mice at 0, 15, 30, 60, and 120 min after intraperitoneal glucose injection when they were 1 year of age. (G) Blood glucose concentrations during ITT in male $F_{1}$ mice at $0,15,30,60$, and 120 min after intraperitoneal insulin injection when they were 45 weeks of age. Data are expressed as mean \pm S.E.M.; ${ }^{\star} P<0.05,{ }^{* \star} P<0.01 \mathrm{DF}_{1}$ vs. DCF . Statistical significance, DF 1 vs. $\mathrm{CCF}_{1}$, is represented using $\dot{X}^{*}$. There is no statistical significance between $\mathrm{DCF}_{1}$ and CCF1. A two-way repeated measurement ANOVA in (A-D,F) and a one way ANOVA in (E), post hoc Bonferroni multiple-

comparisons test was used to assign $P$-values by comparing to each other. $P$-values for significance between groups in repeated measure analysis are shown.

tolerance until 1 year of age. The cause of this different phenotypes between sexes remains to be determined. However, this demonstrated that the impact of uterine environment on offspring's metabolism is more important than gamete alone. Although more and more studies currently tend to suggest that it is the oocytes (Luzzo et al., 2012; Han et al., 2018) or spermatozoa (Wei et al., 2014; Chen et al., 2016; Huypens et al., 2016) of diabetic parents contribute to impact on offspring and emphasize the importance of epigenetic inheritance to increase an offspring's susceptibility to developing obesity and type 2 diabetes. All these studies tried to explain the phenomenon that the prevalence rates for obesity and type 2 diabetes have increased globally over recent decades at a pace by epigenetic inheritance via gametes. As meanwhile, the concern of many researchers, during the last decade, is to explore the physiopathology of the relationship between the health conditions of offspring born from pregnancy complicated with diabetes (Yessoufou and Moutairou, 2011). Offspring developing impaired glucose tolerance or metabolic abnormalities differ according to the level of hyperglycemia during gestation and the period of life studied (Fetita et al., 2006). These studies were carried out in humans (Evers et al., 2004; Atègbo et al., 2006; Grissa et al., 2007; Mitanchez, 2010) and animal models, mostly in rats (Simmons et al., 2001; Lopez-Soldado and Herrera, 2003). As the controversy on the two viewpoints, we first experimentally tested the effect of diabetic uterine environment and gamete by inducing a severe type 2 diabetes mouse model and fertilized egg transfer. As we predicted, uterine environment may play a critical role in increased offspring's susceptibility to obesity or type 2 diabetes. This may contribute to understanding the current obesity and diabetes pandemic.

Overall, we successfully generated a severe type 2 diabetic mouse model to demonstrate that uterine environment of 
maternal diabetes may make a more significant impact on the offspring health compared to epigenetic inheritance via gametes.

\section{DATA AVAILABILITY STATEMENT}

The original contributions presented in the study are included in the article/Supplementary Material, further inquiries can be directed to the corresponding author/s.

\section{ETHICS STATEMENT}

The animal study was reviewed and approved by the Ethics Committee of the Institute of Zoology, Chinese Academy of Sciences. Written informed consent was obtained from the owners for the participation of their animals in this study.

\section{AUTHOR CONTRIBUTIONS}

MZD carried out the analysis, designed the assay, generated mouse models, and wrote the manuscript. QNL, LHF, and LL participated in analyzing the data. ZBW and WS participated

\section{REFERENCES}

Abuaisha, B., Kumar, S., Malik, R., and Boulton, A. J. (1998). Relationship of elevated urinary albumin excretion to components of the metabolic syndrome in non-insulin-dependent diabetes mellitus. Diab. Res. Clin. Pract. 39, 93-99. doi: 10.1016/s0168-8227(97)00111-3

Alberti, K. G., and Zimmet, P. Z. (1998). Definition, diagnosis and classification of diabetes mellitus and its complications. Part 1: diagnosis and classification of diabetes mellitus provisional report of a WHO consultation. Diab. Med. 15, 539-553. doi: 10.1002/(sici)1096-9136(199807)15:7<539::aid$\operatorname{dia} 668>3.0 . c 0 ; 2-\mathrm{s}$

Apelqvist, J., Bakker, K., van Houtum, W. H., Nabuurs-Franssen, M. H., and Schaper, N. C. (2000). International consensus and practical guidelines on the management and the prevention of the diabetic foot. international working group on the diabetic foot. Diab. Metab. Res. Rev. 16(Suppl. 1), S84-S92.

Atègbo, J. M., Grissa, O., Yessoufou, A., Hichami, A., Dramane, K. L., Moutairou, K., et al. (2006). Modulation of adipokines and cytokines in gestational diabetes and macrosomia. J. Clin. Endocrinol. Metab. 91, 4137-4143. doi: 10.1210/jc. 2006-0980

Bayol, S. A., Simbi, B. H., and Stickland, N. C. (2005). A maternal cafeteria diet during gestation and lactation promotes adiposity and impairs skeletal muscle development and metabolism in rat offspring at weaning. J. Physiol. 567(Pt 3), 951-961. doi: 10.1113/jphysiol.2005.088989

Calvo-Maroto, A. M., Perez-Cambrodi, R. J., Albaran-Diego, C., Pons, A., and Cervino, A. (2014). Optical quality of the diabetic eye: a review. Eye (Lond) 28, 1271-1280. doi: 10.1038/eye.2014.176

Chen, Q., Yan, M., Cao, Z., Li, X., Zhang, Y., Shi, J., et al. (2016). Sperm tsRNAs contribute to intergenerational inheritance of an acquired metabolic disorder. Science 351, 397-400. doi: 10.1126/science.aad7977

Cheng, P. P., Xia, J. J., Wang, H. L., Chen, J. B., Wang, F. Y., Zhang, Y., et al. (2011). Islet transplantation reverses the effects of maternal diabetes on mouse oocytes. Reproduction 141, 417-424. doi: 10.1530/rep-10-0370

Chowdhury, J. A., and Nessa, A. (2019). Study on serum total cholesterol, fasting serum glucose, serum glucose 2 hours after meal in Type 2 diabetic patients. Mymensingh. Med. J. 28, 49-53.

Crespi, B. J. (2019). Why and how imprinted genes drive fetal programming. Front. Endocrinol. (Lausanne) 10:940. doi: 10.3389/fendo.2019.00940 in revising the manuscript. QYS designed and revised the manuscript. All authors read and approved the final manuscript.

\section{FUNDING}

This work was supported by a grant from the National Natural Science Foundation of China (31871504) and the High-level Personnel Scientific Research Fund of Qingdao Agricultural University (6651117004).

\section{ACKNOWLEDGMENTS}

We thank all members of the Sun lab for their help and discussion.

\section{SUPPLEMENTARY MATERIAL}

The Supplementary Material for this article can be found online at: https://www.frontiersin.org/articles/10.3389/fcell.2021. 706879/full\#supplementary-material

Evers, I. M., de Valk, H. W., and Visser, G. H. (2004). Risk of complications of pregnancy in women with type 1 diabetes: nationwide prospective study in the Netherlands. BMJ 328:915. doi: 10.1136/bmj.38043.583160.ee

Fetita, L. S., Sobngwi, E., Serradas, P., Calvo, F., and Gautier, J. F. (2006). Consequences of fetal exposure to maternal diabetes in offspring. J. Clin. Endocrinol. Metab. 91, 3718-3724. doi: 10.1210/jc.2006-0624

Filardi, T., Panimolle, F., Crescioli, C., Lenzi, A., and Morano, S. (2019). Gestational diabetes mellitus: the impact of carbohydrate quality in diet. Nutrients 11:1549. doi: 10.3390/nu11071549

Ge, Z. J., Liang, Q. X., Luo, S. M., Wei, Y. C., Han, Z. M., Schatten, H., et al. (2013a). Diabetic uterus environment may play a key role in alterations of DNA methylation of several imprinted genes at mid-gestation in mice. Reprod. Biol. Endocrinol. 11:119. doi: 10.1186/1477-7827-11-119

Ge, Z. J., Liang, X. W., Guo, L., Liang, Q. X., Luo, S. M., Wang, Y. P., et al. (2013b). Maternal diabetes causes alterations of DNA methylation statuses of some imprinted genes in murine oocytes. Biol. Reprod. 88:117. doi: 10.1095/ biolreprod.112.105981

Grissa, O., Ategbo, J. M., Yessoufou, A., Tabka, Z., Miled, A., Jerbi, M., et al. (2007). Antioxidant status and circulating lipids are altered in human gestational diabetes and macrosomia. Transl. Res. 150, 164-171. doi: 10.1016/j.trsl.2007.03. 007

Guariguata, L., Linnenkamp, U., Beagley, J., Whiting, D. R., and Cho, N. H. (2014). Global estimates of the prevalence of hyperglycaemia in pregnancy. Diab. Res. Clin. Pract. 103, 176-185. doi: 10.1016/j.diabres.2013.11.003

Haig, D. (2004). Genomic imprinting and kinship: how good is the evidence? Annu. Rev. Genet. 38, 553-585. doi: 10.1146/annurev.genet.37.110801.142741

Hales, C. N., and Barker, D. J. (2001). The thrifty phenotype hypothesis. Br. Med. Bull. 60, 5-20. doi: 10.1093/bmb/60.1.5

Hales, C. N., and Barker, D. J. (2013). Type 2 (non-insulin-dependent) diabetes mellitus: the thrifty phenotype hypothesis. 1992. Int. J. Epidemiol. 42, 12151222. doi: 10.1093/ije/dyt133

Han, L., Ren, C., Li, L., Li, X., Ge, J., Wang, H., et al. (2018). Embryonic defects induced by maternal obesity in mice derive from Stella insufficiency in oocytes. Nat. Genet. 50, 432-442. doi: 10.1038/s41588-018-0055-6

Hod, M., Kapur, A., and McIntyre, H. D. (2019). Evidence in support of the international association of diabetes in pregnancy study groups' criteria for diagnosing gestational diabetes mellitus worldwide in 2019. Am. J. Obstet. Gynecol. 221, 109-116. doi: 10.1016/j.ajog.2019.01.206 
Huang, Y., Ye, T., Liu, C., Fang, F., Chen, Y., and Dong, Y. (2017). Maternal highfat diet during pregnancy and lactation affects hepatic lipid metabolism in early life of offspring rat. J. Biosci. 42, 311-319. doi: 10.1007/s12038-017-9675-8

Huypens, P., Sass, S., Wu, M., Dyckhoff, D., Tschop, M., Theis, F., et al. (2016). Epigenetic germline inheritance of diet-induced obesity and insulin resistance. Nat. Genet. 48, 497-499. doi: 10.1038/ng.3527

Jayakumari, C., Jabbar, P. K., Soumya, S., Jayakumar, R. V., Das, D. V., Girivishnu, G., et al. (2020). Lipid profile in indian patients with Type 2 diabetes: the scope for atherosclerotic cardiovascular disease risk reduction. Diab. Spectr. 33, 299-306. doi: 10.2337/ds19-0046

Karuranga, S., Malanda, B., Saeedi, P., and Salpea, P. (2019). IDF Diabetes Atlas, 9th Edn. Brussels: International Diabetes Federation.

Laurino, L. F., Viroel, F., Caetano, E. J. M., Spim, S., Pickler, T. B., RosaCastro, R. M., et al. (2019). Lentinus edodes exposure before and after fetus implantation: materno-fetal development in rats with gestational diabetes mellitus. Nutrients 11:2720. doi: 10.3390/nu11112720

Levin, B. E., and Govek, E. (1998). Gestational obesity accentuates obesity in obesity-prone progeny. Am. J. Physiol. 275, R1374-R1379. doi: 10.1152/ajpregu. 1998.275.4.R1374

Lopez-Soldado, I., and Herrera, E. (2003). Different diabetogenic response to moderate doses of streptozotocin in pregnant rats, and its long-term consequences in the offspring. Exp. Diabesity Res. 4, 107-118. doi: 10.1155/edr. 2003.107

Lowe, W. L., Scholtens, D. M. Jr., Lowe, L. P., Kuang, A., Nodzenski, M., Talbot, O., et al. (2018). Association of gestational diabetes with maternal disorders of glucose metabolism and childhood adiposity. JAMA 320, 1005-1016. doi: 10.1001/jama.2018.11628

Luzzo, K. M., Wang, Q., Purcell, S. H., Chi, M., Jimenez, P. T., Grindler, N., et al. (2012). High fat diet induced developmental defects in the mouse: oocyte meiotic aneuploidy and fetal growth retardation/brain defects. PLoS One 7:e49217. doi: 10.1371/journal.pone.0049217

Martino, J., Segura, M. T., García-Valdés, L., Padilla, M. C., Rueda, R., McArdle, H. J., et al. (2018). The impact of maternal pre-pregnancy body weight and gestational diabetes on markers of folate metabolism in the placenta. Nutrients 10:1750. doi: 10.3390/nu10111750

Millership, S. J., Van de Pette, M., and Withers, D. J. (2019). Genomic imprinting and its effects on postnatal growth and adult metabolism. Cell Mol. Life Sci. 76, 4009-4021. doi: 10.1007/s00018-019-03197-z

Mills, J. L., Simpson, J. L., Driscoll, S. G., Jovanovic-Peterson, L., Van Allen, M., Aarons, J. H., et al. (1988). Incidence of spontaneous abortion among normal women and insulin-dependent diabetic women whose pregnancies were identified within 21 days of conception. N. Engl. J. Med. 319, 1617-1623. doi: 10.1056/nejm198812223192501

Mitanchez, D. (2010). [Fetal and neonatal complications of gestational diabetes: perinatal mortality, congenital malformations, macrosomia, shoulder dystocia, birth injuries, neonatal outcomes]. J. Gynecol. Obstet Biol. Reprod. (Paris) 39(8 Suppl. 2), S189-S199. doi: 10.1016/s0368-2315(10) 70046-6

Overland, E. A., Vatten, L. J., and Eskild, A. (2014). Pregnancy week at delivery and the risk of shoulder dystocia: a population study of $2,014,956$ deliveries. BJG 121, 34-41. doi: 10.1111/1471-0528.12427

Pierce, M., Keen, H., and Bradley, C. (1995). Risk of diabetes in offspring of parents with non-insulin-dependent diabetes. Diab. Med. 12, 6-13. doi: 10.1111/j.14645491.1995.tb02054.x

Ratchford, A. M., Chang, A. S., Chi, M. M., Sheridan, R., and Moley, K. H. (2007). Maternal diabetes adversely affects AMP-activated protein kinase activity and cellular metabolism in murine oocytes. Am. J. Physiol. Endocrinol. Metab. 293, E1198-E1206. doi: 10.1152/ajpendo.00097.2007

Saitou, M., Kagiwada, S., and Kurimoto, K. (2012). Epigenetic reprogramming in mouse pre-implantation development and primordial germ cells. Development 139, 15-31. doi: 10.1242/dev.050849

Sasson, I. E., Vitins, A. P., Mainigi, M. A., Moley, K. H., and Simmons, R. A. (2015). Pre-gestational vs gestational exposure to maternal obesity differentially programs the offspring in mice. Diabetologia 58, 615-624. doi: 10.1007/s00125014-3466-7
Schulz, L. C. (2010). The dutch hunger winter and the developmental origins of health and disease. Proc. Natl. Acad. Sci. U S A. 107, 16757-16758. doi: $10.1073 /$ pnas. 1012911107

Simmons, R. A., Templeton, L. J., and Gertz, S. J. (2001). Intrauterine growth retardation leads to the development of type 2 diabetes in the rat. Diabetes 50 , 2279-2286. doi: 10.2337/diabetes.50.10.2279

Simpson, N. E. (1968). Diabetes in the families of diabetics. Can. Med. Assoc. J. 98, 427-432.

Sugiura, K., and Eppig, J. J. (2005). Society for reproductive biology founders' lecture 2005. control of metabolic cooperativity between oocytes and their companion granulosa cells by mouse oocytes. Reprod. Fertil. Dev. 17, 667-674. doi: $10.1071 /$ rd05071

Tan, M. H. (1986). Effect of diabetes mellitus and end-stage renal disease on HDL metabolism. Adv. Exp. Med. Biol. 201, 51-59. doi: 10.1007/978-1-46841262-8_5

Vitoratos, N., Vrachnis, N., Valsamakis, G., Panoulis, K., and Creatsas, G. (2010). Perinatal mortality in diabetic pregnancy. Ann. N Y Acad. Sci. 1205, 94-98. doi: $10.1111 / j .1749-6632.2010 .05670 . \mathrm{x}$

Volpato, G. T., Damasceno, D. C., Sinzato, Y. K., Ribeiro, V. M., Rudge, M. V., and Calderon, I. M. (2015). Oxidative stress status and placental implications in diabetic rats undergoing swimming exercise after embryonic implantation. Reprod. Sci. 22, 602-608. doi: 10.1177/1933719114556485

Vrachnis, N., Antonakopoulos, N., Iliodromiti, Z., Dafopoulos, K., Siristatidis, C., Pappa, K. I., et al. (2012). Impact of maternal diabetes on epigenetic modifications leading to diseases in the offspring. Exp. Diab. Res. 2012:538474. doi: 10.1155/2012/538474

Wang, Q., Frolova, A. I., Purcell, S., Adastra, K., Schoeller, E., Chi, M. M., et al. (2010). Mitochondrial dysfunction and apoptosis in cumulus cells of type I diabetic mice. PLoS One 5:e15901. doi: 10.1371/journal.pone.0015901

Wang, Q., Ratchford, A. M., Chi, M. M., Schoeller, E., Frolova, A., Schedl, T., et al. (2009). Maternal diabetes causes mitochondrial dysfunction and meiotic defects in murine oocytes. Mol. Endocrinol. 23, 1603-1612. doi: 10.1210/me.2009-0033

Wei, Y., Yang, C. R., Wei, Y. P., Zhao, Z. A., Hou, Y., Schatten, H., et al. (2014). Paternally induced transgenerational inheritance of susceptibility to diabetes in mammals. Proc. Natl. Acad. Sci. U S A. 111, 1873-1878. doi: 10.1073/pnas. 1321195111

Yessoufou, A., and Moutairou, K. (2011). Maternal diabetes in pregnancy: early and long-term outcomes on the offspring and the concept of "metabolic memory". Exp. Diab. Res. 2011:218598. doi: 10.1155/2011/218598

Zhang, C. H., Qian, W. P., Qi, S. T., Ge, Z. J., Min, L. J., Zhu, X. L., et al. (2013). Maternal diabetes causes abnormal dynamic changes of endoplasmic reticulum during mouse oocyte maturation and early embryo development. Reprod. Biol. Endocrinol. 11:31. doi: 10.1186/1477-7827-11-31

Zhu, Y., and Zhang, C. (2016). Prevalence of gestational diabetes and risk of progression to Type 2 diabetes: a global perspective. Curr. Diab. Rep. 16:7. doi: $10.1007 / \mathrm{s} 11892-015-0699-\mathrm{x}$

Conflict of Interest: The authors declare that the research was conducted in the absence of any commercial or financial relationships that could be construed as a potential conflict of interest.

The handling editor declared a past co-authorship with one of the author WS.

Publisher's Note: All claims expressed in this article are solely those of the authors and do not necessarily represent those of their affiliated organizations, or those of the publisher, the editors and the reviewers. Any product that may be evaluated in this article, or claim that may be made by its manufacturer, is not guaranteed or endorsed by the publisher.

Copyright (C) 2021 Dong, Li, Fan, Li, Shen, Wang and Sun. This is an open-access article distributed under the terms of the Creative Commons Attribution License (CC BY). The use, distribution or reproduction in other forums is permitted, provided the original author(s) and the copyright owner(s) are credited and that the original publication in this journal is cited, in accordance with accepted academic practice. No use, distribution or reproduction is permitted which does not comply with these terms. 